\title{
Relationship Between Serum Hepcidin and Ferritin Levels in Patients With Thalassemia Major and Intermedia in Southern Iran
}

\author{
Sezaneh Haghpanah ${ }^{1}$; Masoomeh Esmaeilzadeh ${ }^{2}$; Naser Honar ${ }^{2,3}$; Fatemeh Hassani ${ }^{2}$; \\ Javad Dehbozorgian ${ }^{1}$; Narges Rezaei ${ }^{1}$; Maryam Abdollahi ${ }^{2}$; Marzieh Bardestani ${ }^{4}$; Sanaz \\ Safaei ${ }^{1}$; Mehran Karimi ${ }^{1, *}$ \\ ${ }_{2}^{1}$ Hematology Research Center, Shiraz University of Medical Sciences, Shiraz, IR Iran \\ ${ }_{3}^{2}$ Department of Pediatrics, Shiraz University of Medical Sciences, Shiraz, IR Iran \\ ${ }^{3}$ Gastroentology Research Center, Shiraz University of Medical Sciences, Shiraz, IR Iran \\ ${ }^{4}$ Department of Library and Information Sciences, College of Humanities, Khouzestan Science and Research Branch, Islamic Azad University, Ahvaz, IR Iran \\ ${ }^{*}$ Corresponding Author: Mehran Karimi, Hematology Research Center, Nemazee Hospital, Shiraz University of Medical Sciences, Shiraz, IR Iran. Tel/Fax: + 98-7116473239, \\ E-mail: karimim@sums.ac.ir
}

Received: March 1, 2015; Revised: April 8, 2015; Accepted: April 27, 2015

\begin{abstract}
Background: Hepcidin is a key regulator of iron absorption in humans. It is mainly affected by hypoxia and iron stores.
Objectives: The current study aimed to determine the correlation between serum hepcidin and ferritin levels in patients with Thalassemia Major (TM) and Thalassemia Intermedia (TI).

Patients and Methods: The current cross-sectional study investigated 88 randomly selected patients with Thalassemia, 48 TM and 40 TI, registered at the Thalassemia Clinic of Shiraz University of Medical Sciences, a referral center for Thalassemia in Southern Iran in 2013. All patients with TI were receiving Hydroxyurea (HU) $10-15 \mathrm{mg} / \mathrm{kg} /$ day for at least 10 years. The serum hepcidin, ferritin levels, hemoglobin (Hb) and nucleated Red Blood Cell (RBC) of the two groups were measured.

Results: No statistically significant correlation was observed between serum hepcidin and ferritin levels in any of the two groups of patients with $\mathrm{TM}(\mathrm{rs}=0.02, \mathrm{P}=0.892)$ or $\mathrm{TI}(\mathrm{rs}=0.055, \mathrm{P}=0.734)$. The median Interquartile Range $(\mathrm{IQR})$ for serum hepcidin and ferritin levels were significantly higher in TM compared to TI group, (hepcidin: 87.6 (43.9) vs. 51.8 (23.4), P<0.001; ferritin: 2208 (3761) vs. 465 (632), $\mathrm{P}<0.001$ ).

Conclusions: There was insignificant correlation between serum hepcidin and ferritin levels in the two groups of patients with TM and TI. It seems that regulation of hepcidin in patients with Thalassemia is more affected by erythropoeitic activity than iron stores. Also, hepcidin levels were significantly higher in patients with TM than TI, possibly due to higher erythropoeitic activity in TI. In TI, it seems that low dose $\mathrm{HU}$ increases $\mathrm{Hb}$ levels and leads to transfusion-independence, but it is not high enough to suppress bone marrow activity and ineffective erythropoiesis. Consequently, serum hepcidin level decreases.
\end{abstract}

Keywords: Hepcidin; Hydroxyurea; Ferritin; Thalassemia Intermedia; Thalassemia Major

\section{Background}

Beta-Thalassemia is the most common congenital hemolytic anemia characterized by reduction or absence of beta globin chains synthesis resulting in reduction of hemoglobin (Hb) in Red Blood Cells (RBC) and decreased RBC production and anemia $(1,2)$. The regular blood transfusion is known as a basic therapeutic regimen in patients with Thalassemia Major (TM). However, the iron overload in various organs leads to serious problems such as metabolic, endocrine, and growth abnormalities in such patients despite the expanded use of iron-chelator drugs (3-5).

Patients with Thalassemia Intermedia (TI) have a less severe anemia. Although the rate of iron loading is slower in TI than in TM, patients with TI can eventually develop complications similar to those of the patients with TM, including hepatic, endocrine and cardiac dysfunction (6). Moreover, in patients with transfusion independent Thalassemia, serum ferritin was a poor predictor of liver iron concentration; therefore, chelation therapy may be postponed in patients with TI (7).

Hepcidin is a small peptid hormone secreted by hepatocytes to regulate plasma iron concentration and distribution in different tissues $(8,9)$. Hepcidin dysregulation causes a majority of iron related disorders. Chronic excess of hepcidin causes iron restricted anemia (9), while the hepcidin deficiency leads to iron overload with iron deposition in the liver and parenchyma (10). Increased plasma and stored iron stimulate hepcidin production, which in turn blocks dietary iron absorption and consequently iron loading .Conversely, hepcidin is suppressed in iron deficiency (11). Hepcidin is mainly regulated by hypoxia, anemia and iron stores. Hepcidin concentration is significantly higher in chronically transfused patients than in non-transfused ones, presumably due to iron overload (12-16). Despite using iron chelatorin pa-

Copyright (C) 2015, Iranian Red Crescent Medical Journal. This is an open-access article distributed under the terms of the Creative Commons Attribution-NonCommercial 4.0 International License (http://creativecommons.org/licenses/by-nc/4.0/) which permits copy and redistribute the material just in noncommercial usages, provided the original work is properly cited. 
tients with Thalassemia, iron overload is one of the main mortality factors in such patients and hepcidin targeted therapeutics can help with better management of iron overload in patients with Thalassemia (17).

\section{Objectives}

The current study aimed to determine the correlation between serum ferritin and hepcidin levels in patients with TM and TI, also compare serum hepcidin and ferritin levels between the two groups of patients.

\section{Patients and Methods}

The current cross-sectional study was conducted in Hematology Research Center of Shiraz University of Medical Sciences, Shiraz, Iran, in 2013. The study subjects included 90 patients, $50 \mathrm{TM}$ and $40 \mathrm{TI}$, selected by systematic random sampling from the 500 TM and 280 TI patients registered at the Thalassemia Clinic of Tertiary State Hospital affiliated to Shiraz University of Medical Sciences. This is a referral center with 45 beds for patients with Thalassemia in Southern Iran. This study was approved by Medical Ethics Committee of Shiraz University of Medical Sciences (grant number:3652, approval date: 21.03.2011). Informed written consent was obtained from the patients or their parents considering patient privacy.

The diagnosis of Thalassemia was based on Complete Blood Count (CBC), Hb electrophoresis and clinical history. In the TM group, blood transfusion had been started before two years old and all of them were on regular blood transfusion every-two to four weeks. In the TM group, inclusion criteria were age above 10 years old, under regular follow up and regular blood transfusion, and iron chelation therapy. In the TI group, inclusion criteria were transfusion independence and taking hydroxyurea (HU) $10-15 \mathrm{mg} / \mathrm{kg} /$ day for a duration of at least ten years. Patients with the history of any recent infection or surgery, congestive heart and liver failures as well as positive viral hepatitis were excluded from the study. Two patients with TM were excluded from the study based on the aforementioned criteria.

A $2.5 \mathrm{~mL}$ sample of venous blood withdrawn from each participant was added to the tubes containing clot activator. Plasma centrifuge was done immediately and $2 \mathrm{~mL}$ serum was separated and preserved at $-20^{\circ} \mathrm{C}$, then carried by Cold Box to the laboratory to check serum hepcidin and ferritin levels. Also, $2 \mathrm{~mL}$ sample of venous blood was withdrawn from each patient and added to the tubes of $\mathrm{CBC}$ containing ethylenediamine tetraacetic acid EDTA anticoagulant. Complete blood count test was performed immediately with peripheral blood smears and then stained with Wright stain and the rate of Nucleated Red Blood Cells (NRBC) per 100 White Blood Cells (WBC) was counted.

Hemoglobin was measured by Cyanide method, using Sysmex KX21 machine. Fasting serum ferritin was checked by enzyme linked fluorescent assay ELFA method, using Mini Vidas (BioMerievx S.A, France) Machine, and hepcidin was measured by enzyme linked immunosorbent as- say ELISA method (competitive assay), using ELISA Reader dynex (USA) machine with DRG Hepcidin kit (EIA 4 705). According to the direction of hepcidin kit manufacturer, the normal range of hepcidin in $5 \%$ - $95 \%$ of people with the age range of 23 to 67 years is from 13.3 to $54.4 \mathrm{ng} / \mathrm{mL}$. The normal range of ferritin was considered as $20-300 \mu \mathrm{g} /$ Lin males 10 $-100 \mu \mathrm{g} / \mathrm{L}$ in females before menopause, 20 - $200 \mu \mathrm{g} / \mathrm{L}$ in females after menopause and $150-500 \mu \mathrm{g} / \mathrm{L}$ in infants. All of the instruments used in the study were calibrated.

\subsection{Statistical Analysis}

Statistical analyses were performed by SPSS Software version 17.0. Test of normality was done by Shapiro-Wilk test. Among all the evaluated variables, only age had normal distribution. Descriptive data were summarized as mean and standard deviation, as well as median and Interquartile Range (IQR), where appropriate. Correlation between serum hepcidin and quantitative variables in each of the two groups of patients with TM and TI was evaluated by Spearman Correlation Test. Quantitative data between the two groups of patients were compared by Student t-test and Mann-Whitney test. The Chi-square test was used to compare categorical data. A P value < 0.05 was considered statistically significant.

Considering the correlation coefficient of 0.68 for urinary hepcidin and serum ferritin levels reported by Origa et al. (14) in 44 patients with Thalassemia including $22 \mathrm{TM}$ and 22 TI, $\alpha=0.05$ and $\beta=0.01$, the sample size was estimated as 29 patients in each group. For higher accuracy, sample size was increased to $40 \mathrm{TI}$ and $50 \mathrm{TM}$ patients. Two TM patients were excluded from the study.

\section{Results}

The mean age of the patients with TM and TI were 21.4 \pm 6.1 (from 11 to 33 years) and $23.7 \pm 6.1$ years (from 8 to 38 years old) respectively, and there was no statistically significant difference between the two groups ( $\mathrm{P}=0.076)$. Considering gender distributions, 21 (43.8\%) and 22 (55\%) of the subjects in the TM and TI groups were female, respectively; there was no statistically significant difference $(P=0.392)$. The median IQR for serum hepcidin levels were significantly higher in the TM compared to TI group (87.6, 43.9 vs. $51.8,23.4, \mathrm{P}<0.001)$. In addition, the median IQR for serum ferritin levels were 2208 (3761), and 465 (632) in the TM and TI groups respectively; the difference was statistically significant $(\mathrm{P}<0.001)$ (Table 1$)$.

Table 2 indicates the results of correlation between serum hepcidin levels and the evaluated parameters for the subjects with TM and TI. No statistically significant correlations were found between serum hepcidin with any of the evaluated variables in the two groups. Also there were no statistically significant differences in serum hepcidin levels between males and females in patients with TM and TI (P $=0.107$ and $\mathrm{P}=0.861$ respectively). Considering the cut-off point of $54.4 \mathrm{ng} / \mathrm{mL}$ for serum hepcidin levels, 44 (91.7\%), and 18 (45\%) subjects had hepcidin levels higher than normal range in the TM and TI groups, respectively $(\mathrm{P}<0.001)$. 
Haghpanah S et al.

\begin{tabular}{|c|c|c|c|}
\hline Variables & Thalassemia Major $^{\mathrm{C}}$ & Thalassemia Intermedia ${ }^{\mathrm{d}}$ & P Value \\
\hline Age, $y$ & $21.4 \pm 6.1$ & $23.7 \pm 6.1$ & 0.076 \\
\hline Gender & $27 / 21$ & $18 / 22$ & 0.392 \\
\hline Serum ferritin, $\mu \mathrm{g} / \mathbf{L}$ & $2208(3761)$ & $465(632)$ & $<0.001^{\mathrm{e}}$ \\
\hline Serum hepcidin, ng/mL & $87.6(43.9)$ & $51.8(23.4)$ & $<0.001^{\mathrm{e}}$ \\
\hline $\mathbf{H b}, \mathbf{g} / \mathbf{d L}^{\mathrm{f}}$ & $8(1)$ & $9.3(1.5)$ & $<0.001^{\mathrm{e}}$ \\
\hline NRBC per 100 WBC, \% & $3(1)$ & $170(331)$ & $<0.001^{\mathrm{e}}$ \\
\hline
\end{tabular}

a Abbreviations: IQR, interquartile range; SD, standard deviation; NRBC, Nucleated red blood cells; WBC, White blood cell.

b Data are presented as Mean \pm SD, Median (IQR) or male/female.

c Data are presented for $\mathrm{N}=48$.

$\mathrm{d}$ Data are presented for $\mathrm{N}=40$.

e Statistically significant.

$\mathrm{f}$ In Thalassemia major, it was pre-transfusion hemoglobin

Table 2. Correlation Between Hepcidin and Laboratory and Demographic Variables in Patients With Thalassemia Major and Intermedia $^{a}$

\begin{tabular}{lcccccccc}
\hline \multirow{2}{*}{ Group } & \multicolumn{2}{c}{ Ferritin, $\mu$ g/L } & \multicolumn{2}{c}{ Hemoglobin $^{\mathrm{b}}, \mathbf{g} / \mathbf{d L}$} & \multicolumn{2}{c}{ NRBC per 100 WBC, \% } & \multicolumn{2}{c}{ Age, $\mathbf{~}$} \\
\cline { 2 - 9 } & $\mathbf{r}_{\mathrm{S}}$ & P Value & $\mathbf{r}_{\mathrm{S}}$ & P Value & $\mathbf{r}_{\mathrm{S}}$ & P Value & $\mathbf{r}_{\mathrm{S}}$ & PValue \\
\hline TM patients $^{\mathrm{C}}$ & 0.02 & 0.892 & -0.05 & 0.734 & -0.085 & 0.568 & 0.056 & 0.706 \\
TI patients $^{\mathrm{d}}$ & 0.055 & 0.734 & 0.032 & 0.846 & -0.046 & 0.779 & -0.274 & 0.087 \\
\hline
\end{tabular}

a Abbreviations: NRBC, nucleated red blood cells; $r_{s}$, Spearman's rho correlation coefficient; TI, Thalassemia intermedia; TM, Thalassemia major; WBC, white blood cell.

b In Thalassemia major, it was pre-transfusion hemoglobin.

c Data are presented for $\mathrm{N}=48$.

d Data are presented for $\mathrm{N}=40$.

\section{Discussion}

Determining hepcidin concentrations in subjects with iron-loading anemias may be useful to identify the patients at higher risk of iron toxicity due to severely decreased hepcidin levels (17). Based on the current study results, $91.7 \%$ of the subjects with TM had serum hepcidin levels above normal compared to the patients with TI, $45 \%$. Also, the median of serum hepcidin levels were significantly higher in the TM compared to TI group that was similar to those of the previous studies (14). In iron loading anemias not treated by frequent transfusions, such as beta Thalassemia intermedia and congenital dyserythropoietic anemias, urinary and serum hepcidin decrease due to high erythroid signals. Hepcidin deficiency in turn allows excessive iron absorption and development of systemic iron overload $(12-14,18)$. Also, it could be due to more iron overload resulted from regular blood transfusion in the patients with TM compared to TI. As observed in the current study, ferritin levels were significantly higher in the subjects with TM.

Hemoglobin levels were significantly lower in the subjects with TM than in TI, which may be due to the time of blood sampling in the subjects with TM, immediately before transfusion. During transfusion intervals, hemoglobin levels gradually decrease and ineffective erythropoiesis increases.
It is known that in patients with TI, there is also increased risk of iron over load due to increased intestinal iron absorption triggered by chronic anemia, ineffective erythropoiesis and decreased serum hepcidin (19, 20). The current study showed higher erythropoietic activity in the subjects with TI than TM, since the median value for NRBC was significantly higher in TI compared to TM group. In TI group, since all subjects were on HU therapy for a long time, erythropoietic activity was expected to be suppressed and consequently hepcidin level was expected to be higher. But it did not occur and it seems that HU at a dose of $10-15 \mathrm{mg} / \mathrm{kg} /$ day was not enough to suppress bone marrow activity and make erythropoiesis ineffective. However, previous reports documented that HU with the same dose was effective in decrease or cessation of the need for regular blood transfusion, as well as increasing $\mathrm{Hb}$ levels in the patients with Thalassemia (21-24).

The current study showed no significant correlation between serum hepcidin and ferritin levels as a marker of iron overload in any of the TM or TI groups. This result was in accordance with the previous reports that demonstrated the dominant role of erythropoiesis compared to iron overload in regulation of hepcidin in patients with Thalassemia (25-27). In contrast to the results reported by 
Pasricha et al. (15), the current study found no statistically significant correlation between hepcidin and pre-transfusion hemoglobin levels in TM patients. In a longitudinal study on 31 patients with TM, it was documented that transfusion can suppress erythropoiesis and increases hepcidin.

The strong point of the current study was large sample size compared to those of the previous reports; however, there were some limitations in the study, since molecular erythropoietic activity was nit evaluated.

In conclusion, based on the current study results, there was insignificant correlation between serum hepcidin and ferritin levels as a marker of iron overload in any of the two groups of patients with TM and TI, which supported the results of previous studies that regulation of hepcidin in patients with Thalassemia is more affected by erythropoeitic activity than by iron overload. Also serum hepcidin levels were significantly higher in the TM than in TI group, which could mainly result from higher erythropoeitic activity in TI. In the subjects with TI,it is believed that low dose HU (10 - $15 \mathrm{mg} / \mathrm{kg} /$ day) therapy for a long time could increase Hb levels and lead to transfusion-independence, but probably not high enough to suppress bone marrow activity and make erythropoiesisin effective. Consequently, serum hepcidin levels decrease. Increased awareness of hepcidin levels in the patients with Thalassemia could be helpful to diagnose and manage iron burden in such patients.

\section{Acknowledgements}

This article was adopted from Masoomeh Esmaeilzadeh thesis for Specialization in Pediatrics (project number 3652). The authors would like to thank the Research Vice-chancellor of Shiraz University of Medical Sciences, Shiraz, Iran to have financially supported the study. Also, authors wish thank Ms. Shirin Parand at the Hematology Research Center for help in manuscript preparation.Conflict of Interest: The authors report no conflicts of interest. The authors alone are responsible for the content and writing of this article.

\section{Authors' Contributions}

Sezaneh Haghpanah: manuscript editing and statistical analysis; Masoomeh Esmaeilzadeh: manuscript drafting and data collection; Naser Honar and Fatemeh Hassani: data collection; Javad Dehbozorgian and Narges Rezaei: laboratory tests and experiments; Maryam Abdollahi, Marzieh Bardestani and Sanaz Safaei: data collection; Mehran Karimi study design, concept, and manuscript editing.

\section{References}

1. Cao A, Galanello R. Beta-thalassemia. Genet Med. 2010;12(2):61-76.

2. Gardenghi S, Marongiu MF, Ramos P, Guy E, Breda L, Chadburn $A$, et al. Ineffective erythropoiesis in beta-thalassemia is characterized by increased iron absorption mediated by down- regulation of hepcidin and up-regulation of ferroportin. Blood 2007;109(11):5027-35.

3. Aydinok Y, Darcan S, Polat A, Kavakli K, Nigli G, Coker M, et al. Endocrine complications in patients with beta-thalassemia major. JTrop Pediatr. 2002;48(1):50-4.

4. Dubey AP, Parakh A, Dublish S. Current trends in the management of beta thalassemia. Indian J Pediatr. 2008;75(7):739-43.

5. Mashhadi MA, Sepehri Z, Heidari Z, Shirzaee E, Kiani Z. The prevalence of zinc deficiency in patients with thalassemia in South East of iran, sistan and baluchistan province. Iran Red Crescent Med J. 2014;16(8):e6243.

6. Taher A, Isma'eel H, Cappellini MD. Thalassemia intermedia: revisited. Blood Cells Mol Dis. 2006;37(1):12-20.

7. Pakbaz Z, Fischer R, Fung E, Nielsen P, Harmatz P, Vichinsky E. Serum ferritin underestimates liver iron concentration in transfusion independent thalassemia patients as compared to regularly transfused thalassemia and sickle cell patients. Pediatr Blood Cancer. 2007;49(3):329-32.

8. Park $\mathrm{CH}$, Valore EV, Waring AJ, Ganz T. Hepcidin, a urinary antimicrobial peptide synthesized in the liver. $J$ Biol Chem. 2001;276(11):7806-10.

9. Roy CN, Mak HH, Akpan I, Losyev G, Zurakowski D, Andrews NC Hepcidin antimicrobial peptide transgenic mice exhibit features of the anemia of inflammation. Blood. 2007;109(9):4038-44.

10. Roetto A, Papanikolaou G, Politou M, Alberti F, Girelli D, Christakis J, et al. Mutant antimicrobial peptide hepcidin is associated with severe juvenile hemochromatosis. Nat Genet. 2003;33(1):21-2.

11. Ganz T, Olbina G, Girelli D, Nemeth E, Westerman M. Immunoassay for human serum hepcidin. Blood. 2008;112(10):4292-7.

12. Nemeth E, Ganz T. Hepcidin and iron-loading anemias. Haematologica. 2006;91(6):727-32.

13. Kearney SL, Nemeth E, Neufeld EJ, Thapa D, Ganz T, Weinstein DA, et al. Urinary hepcidin in congenital chronic anemias. Pediatr Blood Cancer. 2007;48(1):57-63.

14. Origa R, Galanello R, Ganz T, Giagu N, Maccioni L, Faa G, et al. Liver iron concentrations and urinary hepcidin in beta-thalassemia. Haematologica. 2007;92(5):583-8.

15. Pasricha SR, Frazer DM, Bowden DK, Anderson GJ. Transfusion suppresses erythropoiesis and increases hepcidin in adult patients with beta-thalassemia major: a longitudinal study. Blood. 2013;122(1):124-33.

16. Jenkins ZA, Hagar W, Bowlus CL, Johansson HE, Harmatz P, Vichinsky EP, et al. Iron homeostasis during transfusional iron overload in beta-thalassemia and sickle cell disease: changes in iron regulatory protein, hepcidin, and ferritin expression. Pediatr Hematol Oncol. 2007;24(4):237-43.

17. Nemeth E. Hepcidin in beta-thalassemia. Ann N Y Acad Sci. 2010;1202:31-5.

18. Zhao N, Zhang AS, Enns CA. Iron regulation by hepcidin.J Clin Invest. 2013;123(6):2337-43.

19. Musallam KM, Cappellini MD, Taher AT. Iron overload in betathalassemia intermedia: an emerging concern. Curr Opin Hematol. 2013;20(3):187-92.

20. Musallam KM, Taher AT, Rachmilewitz EA. beta-thalassemia in termedia: a clinical perspective. Cold Spring Harb Perspect Med. 2012;2(7):a013482.

21. Karimi M, Haghpanah S, Farhadi A, Yavarian M. Genotypephenotype relationship of patients with beta-thalassemia taking hydroxyurea: a 13-year experience in Iran. Int J Hematol. 2012;95(1):51-6.

22. Bradai M, Abad MT, Pissard S, Lamraoui F, Skopinski L, de Montalembert M. Hydroxyurea can eliminate transfusion requirements in children with severe beta-thalassemia. Blood. 2003;102(4):1529-30.

23. Ehsani MA, Hedayati-Asl AA, Bagheri A, Zeinali S, Rashidi A. Hy droxyurea-induced hematological response in transfusion-independent beta-thalassemia intermedia: case series and review of literature. Pediatr Hematol Oncol. 2009;26(8):560-5.

24. Yavarian M, Karimi M, Bakker E, Harteveld CL, Giordano PC. Response to hydroxyurea treatment in Iranian transfusion-dependent beta-thalassemia patients. Haematologica. 2004;89(10):1172-8. 


\section{Haghpanah Set al.}

25. Kattamis A, Papassotiriou I, Palaiologou D, Apostolakou F, Galani $\mathrm{A}$, Ladis V, et al. The effects of erythropoetic activity and iron burden on hepcidin expression in patients with thalassemia major. Haematologica. 2006;91(6):809-12.

26. Papanikolaou G, Tzilianos M, Christakis JI, Bogdanos D, Tsimirika $\mathrm{K}$, MacFarlane J, et al. Hepcidin in iron overload disorders. Blood.
2005;105(10):4103-5.

27. Camberlein E, Zanninelli G, Detivaud L, Lizzi AR, Sorrentino F, Vacquer $S$, et al. Anemia in beta-thalassemia patients targets hepatic hepcidin transcript levels independently of iron metabolism genes controlling hepcidin expression. Haematologica. 2008;93(1):111-5. 